\title{
Upper urinary tract pacemaker cells join the GLI club
}

\author{
Doris Herzlinger
}

Department of Physiology and Biophysics and Department of Urology, Cornell University Medical College, New York, New York, USA.

\begin{abstract}
Mutations in GLI3, a component of the Sonic Hedgehog (Shh) signaling pathway, cause a variety of human developmental syndromes. In this issue of the JCI, Cain and colleagues show that tightly regulated GLI3 repressor activity is essential for Shh-dependent differentiation of upper urinary tract pacemaker cells and the efficient flow of urine from the kidney to the bladder. These results link defective pacemaker cell differentiation with hydronephrosis and provide a cellular basis for one of the abnormal renal defects observed in humans with the GLI3-linked disease Pallister-Hall syndrome.
\end{abstract}

\section{The kidney outflow tract is highly prone to congenital defects}

The kidney outflow tract carries wastes removed from the systemic circulation by glomerular filtration and tubular secretion to the bladder (1). It is composed of the renal calyces, pelvis, and ureter and has specialized differentiated properties that mediate this essential conduit function. The outflow tract epithelium has an impermeable luminal surface preventing wastes carried in the urine from diffusing back into the systemic circulation. The proximal-to-distal contractions that propagate through the outflow tract smooth muscle coat are essential for propelling urine into the lower urinary tract.

Despite its seemingly simple structure and function, congenital defects localized to the kidney outflow tract are detected in approximately $0.5 \%$ of fetuses analyzed by routine antenatal sonography (2). These defects obstruct the flow of urine out of the kidney, causing dilations of the renal pelvis (i.e., hydronephrosis). Although a substantial percentage of fetal hydronephrotic syndromes spontaneously resolve, if severe and persistent, hydronephrosis results in permanent kidney damage and is the major cause of renal failure in infants and children.

The causes of congenital hydronephrosis are various (Figure 1). For example, it can be caused by structural occlusions of the outflow tract as a result of compression by ectopic renal vessels, abnormal insertion of

Conflict of interest: The author has declared that no conflict of interest exists.

Citation for this article: J Clin Invest. 2011; 121(3):836-838. doi:10.1172/JCI46400. the ureter into the bladder, or overgrowth of the outflow tract epithelium. Alternatively, it can be caused by functional defects in the formation of the outflow tract smooth muscle coat, leading to severely impaired or even absent peristalsis. Finally, hydronephrosis could be caused by defects in the cells that initiate and coordinate outflow tract contraction.

\section{Elucidating the genes required for outflow tract formation}

Over the past 10 years, several mouse models that develop hydronephrosis during embryonic development have provided insight into the genetic pathways controlling outflow tract formation and function. Targeted deletion of members of the uroplakin gene family, which are selectively expressed by the urothelium, result in structural obstruction as a result of epithelial hyperplasia (3). Proper insertion of the ureter into the bladder wall is essential for the formation of a patent outflow tract, and studies by Mendelsohn's group demonstrated that this process is dependent on retinoic acid signaling mediated in part by the tyrosine receptor cRet (4). Finally, mouse models that develop hydronephrosis in the absence of any obvious structural occlusions in the outflow tract have led to the identification of several signaling and transcription factors required for outflow tract smooth muscle differentiation and organization, including bone morphogenetic protein 4, Tbx18, Teashirt, and Six1, to name only a few (reviewed in ref. 5). However, mice with conditional inactivation of the calcineurin signaling pathway in the periureteral mesenchyme develop hydronephrosis and renal damage despite the presence of a patent outflow tract, differentiated ureteral epithelium, and welldeveloped smooth muscle coat (6).

\section{Pacemaker cells are essential for triggering outflow tract smooth muscle contraction}

Fundamental scientific studies dating back to the late 1800 s provided insight into a possible etiology of hydronephrotic syndromes that could not be explained by either structural occlusion or gross defect in ureteral epithelial or smooth muscle formation. These studies suggested that the efficient flow of urine from the kidney to the bladder is dependent on the presence of specialized myocytes that trigger the coordinated, proximal-to-distal peristaltic waves that propagate through the outflow tract smooth muscle coat (7). These specialized myocytes, called pacemaker cells, elicit spontaneous membrane depolarizations that provide the stimulus for triggering ureteral smooth muscle contraction and play a fundamental role in controlling the frequency and origin of contractile waves in the outflow tract musculature. As in the heart, the outflow tract contains primary pacemakers that trigger contractions at the site of fluid inflow as well as secondary, latent pacemaker activity that can trigger contraction in the event of primary pacemaker failure.

Although the electrophysiological properties of upper urinary tract pacemaker cells have been extensively analyzed, the ion channels and receptors required for their function have remained elusive until recently. Our group has begun to unravel the molecular mechanisms mediating outflow tract pacemaker activity, recently discovering that hyperpolarizing activated cation channels (HCNs) play a fundamental role in triggering outflow tract peristalsis (8). The HCN gene family includes at least 4 members, and $\mathrm{HCN} 2$ and HCN4 are essential for mediating cardiac pacemaker activity (9). We found that HCN3 is selectively expressed by cells adjacent to the renal-most edge of the outflow tract 


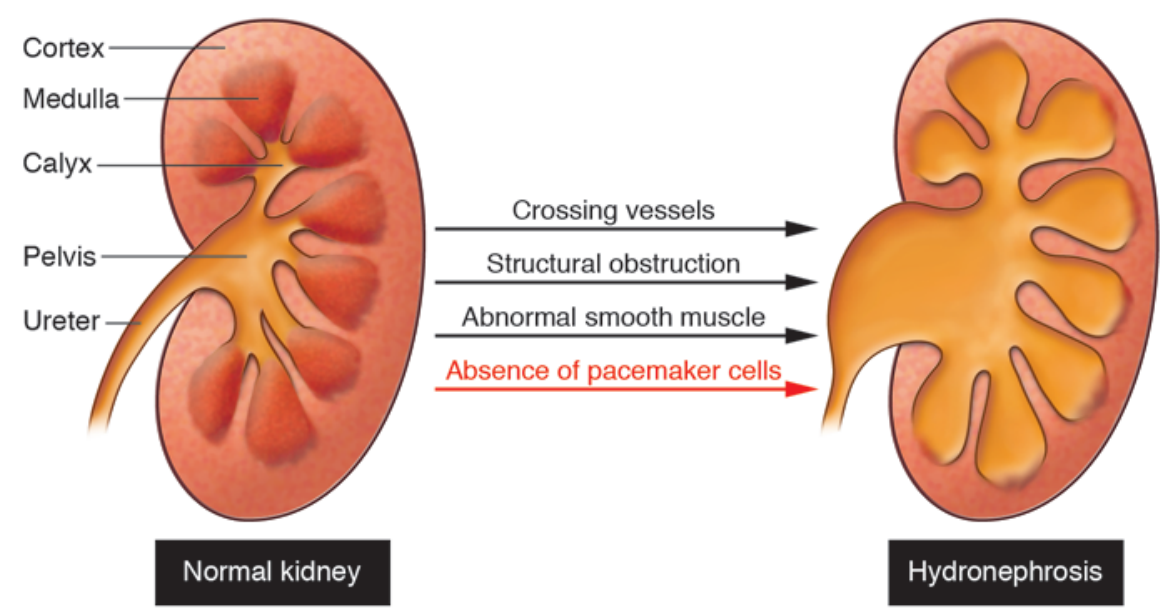

Figure 1

The kidney outflow tract includes the renal calyces, pelvis, and ureter and is highly prone to congenital defects. Abnormalities that severely impair the flow of urine through the outflow tract lead to hydronephrosis and often permanent kidney damage. Known causes of hydronephrosis include outflow tract compression by ectopic renal vessels, structural occlusions intrinsic to the outflow tract, and aberrant outflow tract smooth muscle differentiation that results in aperistaltic ureter segments. The report by Cain et al. (13) shows that hydronephrosis can also be caused by inefficient, dysplastic peristalsis caused by an absence of upper urinary tract pacemakers, the specialized cells that trigger smooth muscle contractions.

musculature, where contractions normally initiate. Furthermore, optical mapping analyses demonstrated that HCN activity is essential for the spontaneous membrane depolarizations that initiate and coordinate outflow tract peristalsis. These data indicate that $\mathrm{HCN} 3$ expression marks primary outflow tract pacemaker cells and that these cells are required for outflow tract function. Moreover, our previous studies indicated that c-Kit tyrosine kinase activity is also required for coordinated outflow tract peristalsis in vitro (10). Cells expressing c-Kit are distinct from $\mathrm{HCN}^{+}$cells and exhibit spatial distribution and electrophysiological properties consistent with secondary outflow tract pacemakers (10-12). Collectively, these results imply that abnormal expression of c-Kit and HCN in the developing outflow tract can lead to uncoordinated and inefficient outflow tract peristalsis and impaired urine flow into the lower urinary tract. The manuscript included in this issue of JCI by Cain et al. provides strong support for this hypothesis (13).

\section{Pacemaker cell differentiation is dependent on regulated Sonic hedgehog signaling}

To understand how abnormal Sonic hedgehog (Shh) signaling in humans causes renal defects, including hydrone- phrosis, Cain et al. disrupted this signaling pathway in the developing murine outflow tract by a variety of genetic manipulations. Previous studies demonstrated that targeted deletion of Shh in the ureter epithelia beginning at early stages of urinary tract morphogenesis resulted in abnormal outflow tract smooth muscle formation (14). The present studies by Cain et al. demonstrate that Shh signaling is also crucial later in development for the acquisition of coordinated outflow tract smooth muscle contraction (13).

Shh signaling is mediated by transcription factors belonging to the GLI family of transcriptional regulators: GLI1, GLI2, and GLI3. Notably, the GLIs variably activate and repress transcription; in the absence of secreted Shh, the cell surface receptor Patched associates with a second protein, Smoothened, converting GLI3 into a transcriptional repressor. In the presence of Shh, Patched dissociates from Smoothened, and GLI3 - along with GLI2 - activates Shh-dependent transcription $(15,16)$.

Cain et al. first showed that disruption of Shh signaling by conditional deletion of Smoothened in the urinary tract mesenchyme at midgestation resulted in hydronephrosis and permanent renal damage (13). Smoothened mutant outflow tracts were patent and surrounded by a contraction- competent smooth muscle coat. However, contractions in this mutant outflow tract musculature were uncoordinated and did not efficiently propel urine from the kidney to the bladder. Further analyses demonstrated that Smoothened-deficient mice exhibited wild-type levels of epithelial and smooth muscle differentiation markers. Strikingly, the mutant mice lacked detectable levels of HCN and c-Kit expression. Collectively, these data indicate that the contraction defects and resulting hydronephrosis observed in Smoothened-deficient mice are caused by abnormal pacemaker cell differentiation.

The above findings, combined with the renal defects observed in humans with Pallister-Hall syndrome, suggest that Smoothened may be required for the upregulated expression of HCN and c-Kit via a GLI3dependent process. To address this, Cain et al. examined outflow tract formation in a murine model of Pallister-Hall syndrome with a targeted mutation in Gli3 $(\Delta 699)$ that generates truncated protein (13). Homozygous Gli3 ${ }^{\Delta 699}$ mutant mice developed urinary tract phenotypes that were nearly identical to those observed in mice with conditional Smoothened inactivation, including an absence of c-Kit- and HCN3expressing cells, uncoordinated peristalsis, and hydronephrosis. Taken together, these data indicate that the acquisition of unidirectional, coordinated peristalsis in the developing outflow tract is dependent on tightly regulated GLI3 repressor activity and provide insight into the etiology of the hydronephrotic syndromes observed in Pallister-Hall patients.

In conclusion, the work of Cain et al. (13), as well as other fundamental scientific studies elucidating the genetic pathways mediating outflow tract formation, provide insight into the etiology of congenital hydronephrosis, one of the most common congenital defects in humans. Results of these studies are likely to lead to novel therapies for the treatment of this condition and provide the groundwork for muchneeded genetic tests that discriminate between fetal hydronephrotic syndromes that spontaneously resolve and those that result in permanent renal damage.

\section{Acknowledgments}

The author thanks Romulo Hurtado, Cindy Liang, and Cathy Mendelsohn for critically reading the manuscript and acknowledges NIH for full support of her research program (grants DK45218 and DK02723). 
Address correspondence to: Doris Herzlinger, Cornell University Medical College, Physiology and Biophysics D-501, 1300 York Ave., New York, New York 10021-4805, USA. Phone: 212.746.6377; Fax: 212.746.8690; E-mail: daherzli@med.cornell.edu.

1. Johnson LR. Essential Medical Physiology. New York, New York, USA: Raven Press; 1992.

2. Becker A, Baum M. Obstructive uropathy. Early Hum Dev. 2006;82(1):15-22.

3. Hu P, et al. Ablation of uroplakin III gene results in small urothelial plaques, urothelial leakage, and vesicoureteral reflux. J Cell Biol. 2000;151(5):961-972.

4. Mendelsohn C. Using mouse models to understand normal and abnormal urogenital tract development. Organogenesis. 2009;5(1):306-314.

5. Airik R, Kispert A. Down the tube of obstructive nephropathies: the importance of tissue inter- actions during ureter development. Kidney Int. 2007;72(12):1459-1467.

6. Chang $\mathrm{CP}$, et al. Calcineurin is required in urinary tract mesenchyme for the development of the pyeloureteral peristaltic machinery J Clin Invest. 2004;113(7):1051-1058.

7. Santicioli P, Maggi CA. Myogenic and neurogenic factors in the control of pyeloureteral motility and ureteral peristalsis. Pharmacol Rev. 1998; 50(4):683-722.

8. Hurtado R, Bub G, Herzlinger D. The pelvis-kidney junction contains $\mathrm{HCN} 3$, a hyperpolarization-activated cation channel that triggers ureter peristalsis. Kidney Int. 2010;77(6):500-508.

9. Biel M, Wahl-Schott C, Michalakis S, Zong X. Hyperpolarization-activated cation channels: from genes to function. Physiol Rev. 2009;89(3):847-885.

10. David SG, Cebrian C, Vaughan ED Jr, Herzlinger D. c-kit and ureteral peristalsis. J Urol. 2005; 173(1):292-295.

11. Pezzone MA, et al. Identification of c-kit-positive cells in the mouse ureter: the interstitial cells of Cajal of the urinary tract. Am J Physiol Renal Physiol. 2003;284(5):F925-F929.

12. Lang RJ, Tonta MA, Zoltkowski BZ, Meeker WF, Wendt I, Parkington HC. Pyeloureteric peristalsis: role of atypical smooth muscle cells and interstitial cells of Cajal-like cells as pacemakers. J Physiol. 2006; 576(pt 3):695-705

13. Cain JE, Islam E, Haxho F, Blake J, Rosenblum ND. GLI3 repressor controls functional development of the mouse ureter. J Clin Invest. 2011; 121(3):1199-1206.

14. Yu J, Carroll TJ, McMahon AP. Sonic hedgehog regulates proliferation and differentiation of mesenchymal cells in the mouse metanephric kidney. Development. 2002;129(22):5301-5312.

15. Biesecker LG. What you can learn from one gene: GLI3. I Med Genet. 2006;43(6):465-469.

16. Riobo NA, Manning DR. Pathways of signal transduction employed by vertebrate Hedgehogs. Biochem J. 2007;403(3):369-379.

\title{
Unraveling virus-induced lymphomagenesis
}

\author{
Chris Boshoff
}

\author{
UCL Cancer Institute, University College London, London, United Kingdom.
}

\begin{abstract}
Kaposi sarcoma herpesvirus (KSHV), a human gammaherpesvirus, is the etiological agent for the endothelial-derived Kaposi sarcoma (KS) and also for certain lymphoproliferative disorders. In these lymphoproliferations, the KSHV-infected cells carry the stigmata of B lymphocytes, with plasmablastic features. The JCI has published three manuscripts addressing key questions related to $B$ cell infection and viral latent expression in B cells. Myoung and Ganem provide evidence that $\mathrm{CD}^{+}$lymphocytes suppress KSHV replication, promoting latency in B cells; Hassman and colleagues show that KSHV infection drives plasmablast differentiation in a subset of $\operatorname{IgM}^{+} \lambda$ light chain-expressing cells; and Ballon and colleagues describe the in vivo transdifferentiation of B lymphocytes by KSHV-encoded viral FLICE-inhibitory protein (vFLIP).
\end{abstract}

Two lymphotropic human herpesviruses are linked to lymphoma development: EBV and Kaposi sarcoma herpesvirus (KSHV). The mechanisms by which EBV infects B lymphocytes and induces their differentiation and proliferation are reasonably well understood (1). In vitro, EBV infection of human primary $B$ cells causes the establishment of latent infection in a fraction of cells exposed to virus, cellular transformation, and the outgrowth of indefinitely proliferating B lymphoblastoid cell lines. In contrast, the lack of B cell systems available for the study of KSHV in vitro and in vivo has hampered our understanding of the natural life cycle of KSHV in B cells and of KSHVinduced B cell lymphoproliferations. The

Conflict of interest: The author has declared that no conflict of interest exists.

Citation for this article: J Clin Invest. 2011; 121(3):838-841. doi:10.1172/JCI46499.
$J C I$ has now published three papers (2-4) that reveal provocative findings regarding KSHV and B cell infection and function.

The main route for infection by EBV and KSHV is via saliva. EBV enters tonsillar B cells via the CD21 receptor and steers the differentiation of pregerminal naive B lymphocytes toward memory cells by way KSHV in saliva (5) and in tonsillar and peripheral CD19+ B cells (6) and the inefficient in vitro infection of primary nonstimulated B lymphocytes from PBMCs prompted the groups of Don Ganem (2) and Dean Kedes (3) to utilize primary tonsillar explants to study KSHV infection ex vivo. Previously, efficient productive or lytic infection of IL-4 and CD40 ligand-activated PBMC-derived B lymphocytes and infection of B lymphocytes from tonsils were demonstrated (7). It is unclear whether activation of B lymphocytes results in the of viral latent transcripts. The presence of upregulation of surface molecules required for KSHV infection, for example, heparin sulfate (8) and DC-SIGN (CD209) (7), and/or whether such activation triggers signaling pathways that encourage viral entry and intracellular transport (9).

Myoung and Ganem showed that exposure of primary human tonsillar explants to KSHV virions results in infection of $\mathrm{B}$ and $\mathrm{T}$ lymphocytes, with B lymphocytes producing substantial amounts of infectious virions (2). Strikingly, and in contrast to exposure of B lymphocytes to EBV, KSHV displays predominantly lytic infection in tonsillar-derived B lymphocytes. This spontaneous lytic viral reactivation of infected B lymphocytes was suppressed when the investigators added activated $\mathrm{T}$ lymphocytes from tonsillar explants. However, these activated $\mathrm{CD} 4^{+} \mathrm{T}$ lymphocytes did not induce B lymphocyte cytolysis and were not dependent on autologous $\mathrm{T}$ lymphocytes being used. Thus, the suppression of spontaneous viral lytic cycle entry in B lymphocytes was MHC unrestricted and not dependent on killing of target cells. Treatment of mixed cultures with the $\mathrm{T}$ cell inhibitor, cyclosporine, abrogated the inhibition of lytic replication. Myoung and Ganem found that activated viable $T$ lymphocytes require physical contact with the infected B lymphocytes to inhibit lytic virus replication. They therefore proposed that unidentified effector $\mathrm{T}$ cell surface ligands are responsible for $\mathrm{T}$ cell-target cell 